\title{
Perspectivas vygotskianas en la educación: el valor cognitivo de la interacción entre iguales*
}

\section{Ellice A. Forman}

\author{
Nortbwestern University
}

\section{Courtney B. Cazden}

\author{
Harvard University \\ Traducción de Laura Pla
}

Dos temas importantes y relacionados en los escritos de Vygotski son los fundamentos sociales del conocimiento y la importancia de la instrucción en el desarrollo:

"Un aspecto importante a señalar en las ideas de Vygotski sobre los orígenes sociales del conocimiento es que ese es el momento a partir del cual empieza a utilizar el concepto de interiorización. No afirma simplemente que la interacción social lleva al niño al desarrollo de sus habilidades en la resolución de problemas, memoria, etc. Más bien dice que los verdaderos medios (especialmente el habla) utilizados en la interacción social son asumidos por el niño e interiorizados. Asi, Vygotski está haciendo aquí una muy seria afirmación acerca de la interiorización y los fundamentos sociales del conocimiento." (Wertsch, 1981, pág. 146.)

«Si todo el desarrollo de la vida mental del niño tiene lugar en el proceso de la interacción social, esto implica que esta interacción y su forma más sistematizada, el proceso de enseñanza, conforma el desarrollo del niño, crea nuevas formaciones mentales y desarrolla procesos mentales superiores. La enseñanza, que a veces parece ir a remolque del desarrollo es, en realidad, su fuerza desencadenante decisiva... La asimilación de la experiencia humana general en el proceso de enseñanza es la forma más importante especificamente humana de desarrollo mental en la ontogénesis.

Esta proposición profundamente significativa define un enfoque esencialmente nuevo para el problema teórico más importante de la psicologia, el reto de desarrollar activamente la mente. La mayor significación de las investigaciones de Vygotski radica en este punto.» (Leontiev y Luria, 1968, pág. 365.)

En todos los escritos de Vygotski que conocemos, la relación social a la cua! se refiere bajo el nombre de «enseñanza» es la relación biunívoca entre un adulto y un niño. Cuando intentamos explorar las perspectivas vygotskianas para la educación, inmediatamente nos enfrentamos a cuestiones sobre el rol del grupo formado por estudiantes como iguales. Aún en el supuesto de que la educación formal se realizara en un contexto de grupo sólo por razones de tipo económico, puesto que no hay sociedad que se pueda permitir el lujo de un profesor por cada niño,

* (Exploring Vygotskian perspectives in education: the cognitive value of peer interaction.) En $\mathrm{J} . \mathrm{V}$. Wertsch (Ed.), Culture, communitation and cognition: Vygotskian perspectives. Nueva York: Cambridge University Press, 1984. Reproducido con autörización. () de esta traducción, Infancia y Aprendizaje, $19^{83}$. 
la presencia de los compañeros no se debería ignorar o relegar a discusiones relativas a temas de gestión y de control de la clase.

Veamos dos aspectos separados pero relacionados relativos a la presencia del grupo. En primer lugar, existen los problemas que se le plantean al profesor al llevar a cabo una enseñanza directa a un grupo de alumnos; en segundo lugar, están las cuestiones relativas a la planificación más indirecta que debe llevar a cabo el profesor para la organización social de todo el discurso relacionado con el trabajo que se produce en el marco de una clase, especialmente la contribución que los compañeros pueden aportarse unos a los otros. En este trabajo nos centramos en este segundo grupo de cuestiones, lo cual no implica una subestimación del primero. Si se concibe la enseñanza como la asistencia al niño en su zona de desarrollo próximo, enseñar a un grupo de niños cuyas "zonas» sólo se superpongan en parte, o no lo hagan en absoluto, plantea problemas obvios. Pero plantear asi los problemas parece básicamente poner nuevas etiquetas al problema, ya familiar, de la variación existente en cualquier grupo que recibe una enseñanza. En cambio, centramos nuestra atención en el problema, menos discutido, de la posible contribución de las interacciones sociales entre los propios niños.

Entender esta contribución tiene una importancia tanto práctica como teórica. En la práctica, a pesar del hecho de que las aulas de las escuelas constituyen unos medios sociales desacostumbradamente poblados, raramente se propicia el trabajo en grupo (Galton, Simon y Croll, 1980), quizá porque no ha habido una clara justificación de su valor. (Ver Sharan, 1980, para una revisión de argumentos y evidencias.) Teóricamente, la mayor parte de los estudios de investigaciones evolutivas realizadas en los Estados Unidos han centrado tradicionalmente su atención en el valor de las interacciones entre iguales para la socialización del comportamiento y la personalidad, tratando menos su posible valor para el aprendizaje intelectual y el conocimiento. Según Lawler ( 1980 ), hasta hace poco tiempo ésta era también la situación en la mayor parte
de los trabajos sobre educación apareci- dos en la Unión Soviética, por ejemplo el trabajo de Makarenko.

Las interacciones entre iguales centradas en el contenido intelectual se pueden situar en un "continuum", de acuerdo con la distribución del conocimiento ò la habilidad entre los niños $y$, por tanto, de acuerdo con los roles que pueden adoptar uno respecto al otro. En un extremo, un niño sabe más que los demás y se espera que actúe de compañero-tutor (o umentor", en el reciente trabajo soviético de M. D. Vinogradova e I. B. Pervin, resumido en Lawler, 1980). En el caso opuesto, el conocimiento es igual, o al menos no intencionadamente desigual, y se espera una colaboración en ambos sentidos y con un "status" igual. En primer lugar, presentamos una investigación sobre dos formas diferentes de tutoria entre companeros $y$, luego, sobre colaboración entre iguales.

Debido a que tanto el análisis empírico como el teórico de las interacciones entre iguales se encuèntra en un nivel tan inicial, incluimos algunos párrafos de protocolos de interacción, no . sólo como evidencia para nuestras interpretaciones, sino también para suministrar material para otras interpretaciones alternativas.

\section{TUTORIA ENTRE COMPAÑEROS}

El informe del discipulo de Vygotski, Levina, señala posibles provechos cognitivos para un tutor a partir de su actividad de dar instrucciones verbales a sus compañeros:

"Vytgotski decia que el habla no incluye en sí misma el poder mágico de crear funcionamiento intelectual. Sólo adquiere esta capacidad al utilizarse en su capacidad instrumental» (Levina, I981, pág. 296).

Suponiendo que esto sea cierto, lo que Levina llama la «intelectualización», así como la interiorización del lenguaje, debería resultar favorecida con la utilización del lenguaje instrumental con otros. Levina sugire exactamete esto:

"Lo que se percibe en silencio como algo unitario y completo se disgrega inmediatamene en sus componentes en cualquier intento de formularlo verbal- 
mente. Es fácil convencerse de esto cuando se intenta introducir la claridad de una formulación verbal a una impresión inconsciente. ¿Cuáles son las fuerzas motivadoras que sustentan este tipo de formulación verbal? ¿Qué es lo que impulsa al niño a representar sus percepciones verbalmente $y$ formular $y$ etiquetar sus acciones? Al contestar a estas pregunts, Vygotski puso un especial énfasis en los factores relacionados con el orden social. Pensaba que el niño, al poner una etiqueta a una acción en curso, está en un principio, teniendo en cuenta a las personas que le rodean a través de la representación verbal. Les hace accesibles esta representación, como si se la quisiese clarificar. Vygotski creía que el simple acto de poner etiquetas surgia de la necesidad de conferir a las propias acciones, una forma especializada comprensible para los denás» (Leviña, igoôi, págs. żo-g).

Los ejemplos de Levina de este etiquetado, que la necesidad de comunicarse con otros estimula, tomados de las notas y protocolos recogidos bajo la supervisión de Vytgotski, contienen sólo el lenguaje de niños dirigido al experimentador. Si bien no se habla de la necesidad de comunicarse con los compañeros, parece consistente con la perspectiva de Levina/Vytgotski que la necesidad de comunicarse con un compañero con menos conocimientos - tal como un compañero de la clase- originaría un proceso idéntico, por lo menos con la misma fuerza. A este respecto, la instrucción de los compañeros podria suponer un paso intermedio entre la situación de ser dirigido receptivamente por el lenguaje de otro y la de dirigir productivamente el propio proceso mental mediante el lenguaje interior.

El primer análisis de la tutoria entre compañeros viene de una investigación llevada a cabo en un aula primaria de San Diego. Se grabaron en video doce secuencias de tutoría entre compañeos llamadas cadenas instruccionales (ICs), para ser posteriormente analizadas (Cazden, et al., 1978; Carrasco, Vera y Cazden, en prensa). Resumiendo, en cada IC el profesor $(P)$ enseñaba una lección a un niño, el cual enseñaba luego la misma lección a uno o más compañeros. Leola, una alumna negra de tercer grado, recibió la tarea de aprender y luego enseñar una taréa de lenguaje. Aqui están los tres primeros items de su hoja de trabajo, en su forma completa*.

$$
\begin{aligned}
& \text { 1. Nuevo } \\
& \text { 2. No } \\
& \text { 3. Fuera me lo dijiste }
\end{aligned}
$$

En la página siguiente hay una transcripción, exceptuando las repeticiones, correcciones, etc., de las indicaciones del profesor mientras enseñaba a I.eola cómo realizar los dos primeros items del trabajo.

Nótese, en primer lugar, que las preguntas del profesor sirven para acompañar a Leola a través del trabajo hasta que lo sabe hacer sola, proceso que ha mostrado Wertsch (1978) en relación con la ayuda de las madres a sus hijos de preescolar en la tarea de realizar un rompecabezas. El

\begin{tabular}{|c|c|}
\hline Profesor & Leola \\
\hline 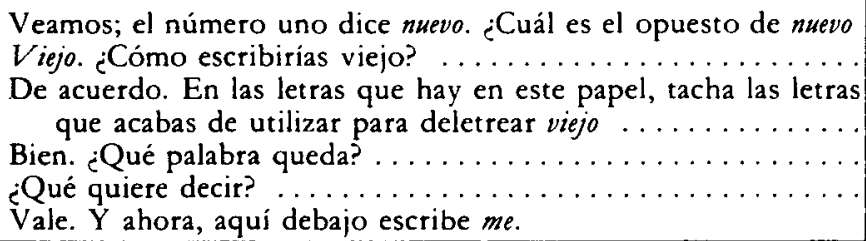 & $\begin{array}{l}\text { Viejo } \\
\text { V-I-E-J-O } \\
\text { (L., lo hace) } \\
\mathrm{M}-\mathrm{E} \\
\mathrm{Me}\end{array}$ \\
\hline \multicolumn{2}{|l|}{ Item 2.} \\
\hline 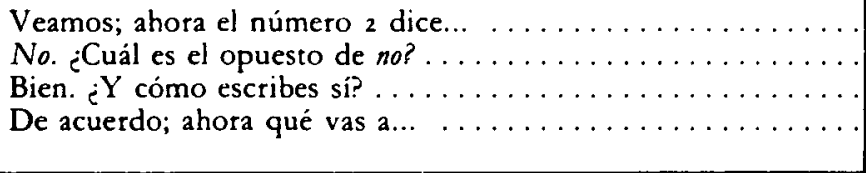 & $\begin{array}{l}\text { No } \\
\text { Si. } \\
\text { S-I } \\
\text { (L tacha las letras S-I) } \\
\text { LO }\end{array}$ \\
\hline
\end{tabular}
hecho de que una ayuda de este tipo

Item I

* N. del T. Adaptación al castellano del original. Iaas diferencias sintácticas impedian su traducción directa. Fl original dice así:

$\begin{array}{lllllll}\text { 1. new } & \text { 1. } & \text { Y } \phi \text { hodu } & \text { 2. } & \text { tyfolpd } & \text { 3. m mple } \\ \text { 2. no } & & \text { You } & \text { told } & \text { me } \\ \text { 3. off } & & & & & \end{array}$


permita que luego Leola trabaje independientemente se observa comparando las instrucciones del profesor en el primer y segundo ítem. Se repiten las tres primeras preguntas, pero luego la pregunta mucho más vaga e incompleta de "y ahora qué vas a", es suficiente y Leola sigue por su cuenta.

El segundo aspecto notorio de este IC desde la perspectiva de Levina/Vygotski es el desarrollo de una mejor articulación y precisión en las verbalizaciones de Leola a lo largo de la tarea. Si se considera toda la cadena instructiva como un «test de imitación del discurso", entonces las instrucciones deben ser reconstruidas mediante el sistema cognitivo, lingüístico y sociolingüístico del tutor. Si bien el profesor enseñaba con preguntas, Leola enseña con afirmaciones, a menudo "debes X» Mehan y Riel (en prensa), muestran que este contraste en los estilos de enseñanza era característico de los 12 IC.

A Leola no le fue fácil, de forma inmediata, traducir a palabras las indicaciones para esta tarea. Cuando, al principio, Leola intentó explicar al profesor, tarea previa, qué era lo que iba a decir a su grupo, incluyó sólo la referencia explícita de uno de los cuatro componentes esenciales, la idea de "dejar" algunas letras:

P: Dime qué les vas a decir que hagan.

L: Deletrear estas letras y luego quitar esa letra, y, luego, dejar otra letra fúera.

El profesor repite las instrucciones, y esta vez pide a Leola especificamente que repita la palabra «opuesto". Entonces, Leola, incluye dicha palabra, pero con el verbo "hacer», de significado vago:

P: Quieres tachar el opuesto de «nuevon. Es mejor que lo digas, porque va a ser realmente importante. Ellos van a leer "nuevo" y luego, ¿qué es lo que van a hacer?

L: Harán lo opuesto.

Leola consigue la explicación más clara en el tercer intento (sin dudas ni autocorrecciones):

El opuesto de fuera es dentro, así, en el número 3 debes tachar dentro D-EN-T-R-O, y queda dijiste D-I-J-I-S-T-E.

En general, uno está tentado a argüir I 42 que el cambio en las instrucciones de
Leola constituye un ejemplo de lo que Wertsch y Stone (2978), siguiendo a los psicólogos soviéticos, llaman microgénesis -es decir, desarrollo dentro de un periodo de tiempo observable-, y es el tipo de desarrollo que parecía necesitar Leola. En las nueve lecciones analizadas por Mehan (1979), unas tres horas de charla en total, Leola habló cuarro veces, y sólo en dos ocasiones más de una palabra. Esto no quiere decir que fuese en ningún sentido no-verbal; pero sugiere que podría sacar provecho de retos para formular contenidos académicos en palabras, y que las exigencias de hacer de tutor, incluyendo la necesidad de formulaciones repetidas y correcciones a los demás, suponen muy bien un reto de este tipo. Si existe validez en la hipótesis de la interiorización, la práctica realizada en formulaciones explicitas debería, en último extremo, ayudar también al lenguaje interior. Un lenguaje vago, no explicito - o una percepción unitaria y no formulada, con palabras de Levina - no es lo mismo que la predicación y el "sentido" en el lenguaje interior.

Finalmente, se da una interesante reducción de información en las instrucciones de Leola después del item 3. Con dos excepciones, en todos los items posteriores al 3, Leola habla en voz alta, con la cabeza baja, mientras cumple con su tarea. En los casos reducidos 4-s y 7-10, la reducción en la información se debe más a formulaciones alternativas de los componentes que a su olvido. Por ejemplo, la palabra crítica «opuesto" sólo aparece en los casos i a 3, cuando se debe repetir el primer trabajo (i R) y en el caso 6. En los otros casos, Leola se limita a decir "fuera es dentro" (presuponiendo que es significa es el opuesto de) o, de forma incluso más breve, yuxtaponiendo simplemente las dos palabras "oeste este». En las dos excepciones, IR y 6, Leola vuelve a ser explícita. cuando corrige a sus tutorandos al darse cuenta de que han cometido un error.

Existen dos posibles explicaciones alternativas para la menor explicitación en los casos reducidos. Se puede deber, bien a la comprensión de Leola de que el concepto de "opuestos» se puede ya suponer, o a la menor explicitación que caracteriza al lenguaje para uno mismo. Según señala Wertsch (1979), la descomposición de la información vieja o «dada» 
es funcionalmente equivalente en el diálogo y en el lenguaje privado.

El segundo análisis de la tutoria entre compañeros, proviene de las observaciones realizadas por Kamler (1980) en un aula de segundo grado de New Hampshire, en la cual el equipo de investigación de Donald Graves estaba observando la enseñanza de la escritura. La profesora Egan mantenía regularmente conversaciones con los niños por separado. Además, animaba a los niños a que tuviesen "conversaciones entre compañeros» acerca de sus escritos. A continuación, presentamos el informe de un observador de una conversación mantenida entre dos niños, Jill y Debbie:

«El 1 de marzo, Jill era uno de los seis niños citados para una entrevista sobre escritura... Bajo la dirección de Egan, Jill y los otros citados se acercaron a la mesa de lenguaje. Egan habia pedido a Jill que, primero, pasase un rato con Debbie, de siete años, revisando el texto para estar segura de que estaba lista para la entrevista...

Jill comenzó leyendo a Debbie cada página en voz alta... Mientras Jill escuchaba sus propias palabras, introdujo cambios en las páginas $1,{ }_{2}$ y 3 sin indicación o comentario alguno por parte de Debbie, y, en las páginas 4, 5 y 8 como respuesta directa a preguntas planteadas por Debbie...

Al finalizar esta entrevista de media hora, Jill habia introducido seis cambios que afectaban al sentido global del texto. Habia eliminado la información sin sentido o que no podía justificar; añadió información para clarificar o explicar. La presencia de Debbie èra crucial para las revisiones de contenido. Su presencia física obligó a jill a releer el texto por primera vez desde su composición; parecía como si Debbie le hiciese visible el concepto de audiencia. Jill necesitaba también un lector activo para hacer preguntas...

(Más tarde), Debbie reclamó su turno: " $V$ Vale, Jill, ahora me ayudas a mí!". Invirtieron los roles, y volvieron de nuevo a la mesa de lenguaje para trabajar en el texto de Debbie, Ice Follies, hasta que la señorita Egan estuvo dispuesta para ver a Jill, veinte minutos más tarde». (Kamler, 1980, págs. 683-5).

En primer lugar, cabe destacar que, en este caso, se trata de un modelo más reciproco de ayuda entre compañeros. Los roles de escritor e interrogador son intercambiables entre los niños. Todos los niños pueden aprender qué hacer y decir en el rol de interrogador a partir del modelo del profesor en la entrevista que mantiene con él, un modelo consistente de cómo plantear preguntas que sirvan de ayuda centradas en el contenido del escrito, no en su forma. El profesor cree que las preguntas centradas en el contenido son de mayor ayuda que las preguntas acerca de la forma; por otro lado, son también el tipo de preguntas que los niños se pueden hacer con sentido unos a otros. Así, el modelo del profesor posibilita que los niños intercambien entre si el rol de profesor, lo que beneficia al niño que esté ejerciendo de autor, que podrả adquirir tantas más experiencias como responsiva sea su audiencia; y beneficia, tambièn, al niño que ejerce de critico, que podrá interiorizar tales preguntas mediante el proceso que implica el hecho de no limitarse sólo a responder al profesor, sino de plantearlas también a sus compañeros.

Para que estas ventajas se hagan realidad, el modelo del profesor debe ser aprehensible por los niños. Graves informa (comunicación personal, 1980) que la estructura de la entrevista empleada por otro profesor de la misma escuela les resultaba más difícil de aprender a los niños, por lo que el efecto multiplicador de las entrevistas entre compañeros era menor en su clase. Esta comparación sugiere que, en una clase, el valor intelectual de las interacciones entre compañeros aumentará cuando el profesor modele consistentemente un tipo de interacción que los niños puedan aprender para utilizar al hablar entre ellos.

Tal como señala Kamler, el niño escritor se beneficia en dos maneras diferentes de la presencia del compañero. La más obvia, el compañero hace preguntas siguiendo el modelo adulto, pero con contenidos apropiados al escrito en cuestión; y algunos de los cambios de Jill (págs. 4, s y 8) eran una respuesta directa a las preguntas formuladas por Debbie. De manera menos obvia, en silencio, pero no por ello menos eficazmente, el compañero representa las necesidades de una audiencia, y hace «visible el concepto de audiencia».

Podemos situar el efecto de un auditorio silencioso de este estilo en la casilla 
de otro modo vacía, creada por la distinción que Wertsch y Stone (este volumen, págs. I I-I 2) hacen de las dimensiones social/individual y externa/interna en el análisis de Vygotski. Wertsch y Stone separan las dos dimensiones con el fin de hacer un lugar al lenguaje egocéntrico, que es externo en la forma, pero indivi- dual en cuanto a su función. Sugerimos que los cambios que introduce Jill como respuesta a la presencia silenciosa de Debbie, son exactamente lo opuesto: internos en la forma ( $\mathrm{si}$ bien reflejados en lo escrito) y sociales en cuanto a su función, de modo que el escrito resulte más informativo para los demás.

\begin{tabular}{|l|l|l|}
\hline & \multicolumn{1}{|c|}{ Externo } & \multicolumn{1}{|c|}{ Interno } \\
\hline Social $\ldots . . .$. & Lenguaje totalmente comunicativo. & $\begin{array}{l}\text { Redacción silenciosia. } \\
\text { Lenguaje interiorizado. }\end{array}$ \\
\hline
\end{tabular}

En palabras de A. N. Leontiev, «al proceso de interiorización no es la transferencia de toda la actividad externa a un "plano de consciencia" interior preexistente, es el proceso en el cual se forma este plano interiorn (1981, pág. 57 , subrayado en el original). El concepto de un estadio intermedio favorable entre lo completamente externo y lo completamente interiorizado, existe ya en la psicología soviética. Slavina (1957) suministra un ejemplo análogo en el desarrollo que va desde contar objetos hasta la aritmética mental: cuando "Voya» podía realizar sumas simples, si tenía objetos para manipular, pero no las podia realizar mentalmente, se introdujo un estadio intermedio: Después de manipular los objetos y contar en voz alta, se pidió a Voya que realizase el mismo cálculo en voz alta, pero sin mirar a los objetos que seguían delante suyo. Slavina encontró que esta actividad intermedia parecía ayudar a los niños a formarse una representación mental de los objetos, colaborando en su progreso hacia un cálculo totalmente encubierto?

\section{COLABORACION ENTRE IGUALES}

Si se compara con la tutoría entre compañeros, es todavia menos conocido el valor intelectual de la colaboración entre iguales. Puede que esto se deba, en parte, a que la colaboración exige un entorno de trabajo que está muy lejos del aula tradicional. Las tareas realizadas en régimen de tutoría entre compañeros, tienden a parecerse a las actividades comunes en las aulas: rellenar los libros de ejercicios, leer en voz alta, redactar ejer- cicios escritos, etc. En estas actividades, el tutor ayuda al tutorado informándole, guiándole y/o corrigiéndole. La colaboración exige una tarea mutua, en la cual los compañeros trabajan juntos para producir algo que ninguno habría podido producir por su cuenta. Dado el enfoque de logro individual existente en la mayoria de las sociedades industriales pocas veces se encuentran en las escuelas planificaciones que promuevan la colaboración ni éstas son estudiadas por los educadores o los psicólogos.

Por esta razón, la investigación acerca de la cooperación entre iguales ha sido escasa. La excepción más importante a esta situación general la constituye un bloque de investigación dirigido por un grupo de psicólogos ginebrinos (Doise, Mugny y Perret-Clermont, 1975, 1976; Mugny y Doise, 1978; Perret-Clermont, 1980), que han llevado a cabo una serie de experimentos para examinar el efecto de la colaboración entre iguales sobre el razonamiento lógico asociado con el estadio piagetiano de las operaciones concretas: cambio de perspectiva, conservación, etcétera.

La mayor parte de las investigaciones de Ginebra ejemplean un diseño de entrenamiento en el que los sujetos son asignados al azar a unos grupos de tratamiento o control, en los cuales son expuestos a diferentes contextos sociales. Por ejemplo, los sujetos en el grupo de tratamiento pueden recibir el encargo de resolver una tarea de conservación en el marco de un pequeño grupo de compañeros compuesto por conservadores y no conservadores, mientras que los sujetos del grupo control deben resolver el mismo problema por sí solos. Todos los sujetos pasan 
individualmente un pretest y un postest con una tarea estándar de razonamiento concreto-operacional, y el efecto de la exposición a la colaboración entre iguales se verifica comparando el avance observado entre el pretest y el postest en ambos grupos en cuanto al razonamiento concreto-opéracional. Los psicólogos ginebrinos han empleado este mismo diseno en toda una serie de estudios en los que las tareas específicas de razonamiento, los grupos sociales reunidos y los criterios utilizados para evaluar el crecimiento cognitivo varian de forma sistemática. Tras revisar todo este cuerpo de investigación, Perret-Clermont (1980) llega a la conclusión de que la interacción entre compañeros aumenta el desarrollo del razonamiento lógico a través de un proceso de reorganización cognitiva activa inducida por el conflicto cognitivo ( 1980 ). Afirma que el conflicto cognitivo aparece con mayor facilidad en aquellas situaciones en las cuales se les pide a unos niños con perspectiva moderadamente discrepantes (por ejemplo sujetos conservadores $y$ transicionales) que alcancen un consenso.

Dos investigadores rusos, Lomov (1978) y Kolitsova (1978), y dos japoneses, Inagaki y Hatano (1968, 1977, 1981) han llegado a conclusiones similares: que la interacción entre compañeros ayuda a mejorar el conocimiento individual e integra una variedad de perspectivas relativas a un problema, y que este proceso de coordinación, por su parte, produce unos resultados intelectuales superiores. Para Kolitsova, los resultados son definiciones precisas, ricas y lógicamente rigurosas de un concepto de ciencia social. Para Inagaki y Hatano, los resultados son conceptos generalizables y estables de conservación. Para Perret-Clermont, los resultados son un aumento de la habilidad para utilizar una lógica operacional concreta.

En ninguno de estos estudios se observaron, sistemáticamente, las interacciones de los sujetos durante la solución del problema en colaboración. Sólo existe una evidencia anecdótica para apoyar la hipótesis de que la interacción entre compañeros puede mejorar la realización intelectual personal, porque obliga a los individuos a reconocer $y$ coordinar perspectivas conflictivas sobre un problema. Para comprobar esta hipótesis, seria necesario examinar el proceso de coordinación social que se produce durante la solución del problema con el fin de aislar las condiciones sociales más responsables dél crecimiento cognitivo. Por ejemplo, se podrian observar las interacciones que se producen mientras el grupo trabaja con el fin de diferenciar aquellos grupos en los cuales los miembros trabajan en estrecha relación e intentan coordinar frecuentemente sus perspectivas, de aquellos en los cuales los miembros trabajan en gran parte por su cuenta. A continuación, se podría examinar cómo afectan estos modelos de interacción en grupo a las estrategias utilizadas para la resolución del problema. Este es, precisamente, el enfoque propuesto por Perret-Clermont:

"Hemos observado que para que la tarea tenga un valor educativo no es suficiente con dar a los niños una tarea común; debe producirse también la confrontación entre distintos puntos de vista. ¿Son todas las actividades descritas como "cooperación" por los investigadores de naturaleza tal que induzcan a coordinaciones reales interindividuales, las cuales son la fuente de conflicto cognitivo? Esta pregunta sólo puede responderse mediante una observación sistemática, que está aún pendiente de realización..." (1980, pág. 196).

«En otros estudios de la psicologia de la inteligencia deberiamos tener en cuenta no sólo el efecto de la coordinación interindividual sobre el comportamiento de juicio, o sobre la realización como indice de desarrollo..., sino tambin el impacto de diferentes tipos de interacción social y, en particular, de las estrategias de los compañeros, sobre la estrategia adoptada por el sujeto para cumplir la tarea..." (1980, pág. 192).

Describiremos un estudio que acaba de ser terminado (Forman, 198I) en el cual se analizaron grabaciones en vídeo de sesiones de solución de problemas en régimen de colaboración con el fín de analizar los modelos de interacción social utilizados y las estrategias de solución de problemas empleadas. Además, de esta muestra de sujetos que habian resuelto los problemas en régimen de colaboración, se recogieron mediciones individuales de razonamiento lógico, comparándolas con mediciones similares de sujetos que habian resuelto los problemas individualmente recogidos en un muestreo previo. 
El diseño de la investigación utilizado por Forman es una modificación del diseño de entrenamiento utilizado por Perret-Clermont y sus colegas. En lugar de suministrar a los niños sólo una oportunidad de solucionar un problema en régimen de colaboración, Forman expuso a sus sujetos a un total de once sesiones de solución del problema. Existen varias razones para utilizar un diseño longitudinal con el fin de evaluar las habilidades de los niños en la solución de problemas. Se puede observar el proceso de crecimiento cognitivo directamente, en vez de inferirlo a partir de la realización en el pretest y postest, y los niños pueden desarrollar relaciones de trabajo estables. Además, se eligió un diseño longitudinal para este estudio, a fin de que los datos obtenidos con la resolución colectiva de problemas se pudiesen comparar con los datos longitudinales similares obtenidos por Kuhn y Ho (1980) en la resolución de problemas en régimen individual. (Ver Kuhn y Phelps, 1979, y Forman, 1981, para una explicación más detallada de los puntos clave de este tipo de diseño longitudinal.)

Así, el estudio de Forman suministra dos tipos de informaciones acerca de la colaboración: cómo las estrategias de razonamiento de los sujetos que resuelven los problemas en régimen de colaboración difieren de aquellos que lo hacen en régimen individual, y cómo algunos grupos difieren de otros tanto en los modelos de interacción social como en la estrategia cognitiva empleada. En la discusión que sigue a continuación nos centraremos en estos dos tipos de datos: comparación de colaboradores con los sujetos que trabajan en régimen individual y comparación entre diferentes grupos que trabajen en régimen de colaboración. Acto seguido discutiremos los hallazgos de Forman a la luz de las hipótesis de PerretClermont y de la teoria, a nuestro juicio esencial y complementaria; de Vygotski.

\section{EL ESTUDIO DE FORMAN}

Al igual que Perret-Clermont, Forman pidió a los niños que cooperasen en la solución de una tarea piagetiana de razonamiento lógico. En contraste con Perret-Clermont, Forman seleccionó una tarea utilizada para verificar el razona- miento operacional formal, un problema químico de combinación. Además, sus sujetos eran mayores (aproximadamente, unos nueve años) que los seleccionados por Perret-Clermont (cuatro-siete años).

En los dos estudios realizados por Forman (1981) y Kuhn y Ho (1980) los sujetos eran de cuarto-quinto grado, de clase media - Is individuos solos (Kuhn y Ho) y cuatro parejas (Forman) y no mostraban ninguna habilidad para aislar variables en una tarea formal (simple planteamiento del problema). Junto con el pretest utilizado para la selección, todos los sujetos recibieron un pretest adicional: un problema de combinación en el cual se les pedía ordenar cinco tipos de aperitivos en todas las combinaciones posibles. Tanto los sujetos individuales como las parejas participaron en i i sesiones de solución de problemas, aproximadamente, una vez por semana a lo largo de un período de tres meses. Las dos mediciones del pretest se readministraron como postest dentro de la semana siguiente a la finalización de las sesiones de solución de problemas. Todos los pretest y postest se administraron a nivel individual.

El problema de combinación química era una adaptación de una tarea de Inhelder y Piaget conocida como combinaciones de cuerpos químicos con color e incoloros (1958), consistente en una serie de siete problemas ordenados en términos de complejidad lógica. El problema I, el más sencillo, exige que los sujetos identifiquen el producto químico que, de un conjunto de cinco productos inodoros e incoloros, es necesario y suficiente para producir un cambio de color específico cuando es mezclado con un reactivo. En los problemas 2 y 3, dos o tres de los cinco productos químicos pueden producir el cambio de color, bien por separado o juntos. En el problema 4, dos productos químicos son capaces de producir el cambio, pero sólo cuando ambos están presentes, etcétera.

El problema I, con un producto químico operativo diferente cada vez, fue presentado durante las cuatro primeras sesiones. Este procedimiento garantizaba la exposición repetida de los niños al problema más sencillo de la serie antes de que se introdujesen problemas más difíciles. Tras la cuarta sesión se presentaba un 
nuevo problema de la serie cada vez que se resolvía el anterior. Así, el progreso a través de la serie de problemas es una medida de la efectividad de las estrategias empleadas por el (o los) sujetos en la resolución del problema.

Cada una de las once sesiones experimentales siguió el mismo esquema de ambos estudios. En primer lugar, el experimentador realizaba dos experimentos de demostración. Seguidamente, formulaba un conjunto estándar de preguntas a los niños acerca de la demostración, como por ejemplo: “¿Qué crees que hace que se vuelva morado.» Luego pedía a los niños que dispusiesen los experimentos que querian intentar para determinar qué producto(s) químico(s) eran responsables del cambio. Durante esta fase de preparación no se permitía la mezcla de los productos químicos. Una vez que los experimentos estaban preparados, y tras formular algunas preguntas más, se permitía a los niños realizar las combinaciones seleccionadas. En el estudio de Forman se animaba a las parejas a que trabajasen juntas en la preparación y ejecución de los experimentos. Finalmente, una vez observados los resultados de las combinaciones, el experimentador repetía el conjunto original de preguntas para verificar si se habia identificado el producto (o productos) químico correcto.

Forman analizó sólo la parte de las sesiones destinada a planificar y distribuir los experimentos. Se codificaron cuatro sesiones para tres parejas de sujetos (George y Bruce: sesiones \# 3, \# 5 , \# 8, \# I I; Lisa y Linda: sesiones \#3, \#:5, \# 9, \#: I I; Matt y Mitch: sesiones \#3, \# 5, \# 8, \# IO; un total de 12 cintas. (La cuarta pareja se había introducido sólo como garantía frente a una posible enfermedad, etcétera.) Los dos sistemas de código utilizados en el análisis consistieron en un conjunto de categorías de interacción social y en un conjunto de categorias de experimentación. En este trabajo sólo vamos a discutir un tipo de código de comportamiento social (interacciones de procedimiento) y tres tipos de estrategias de experimentación (azar, aislamiento de variables y combinatoria).

Las interacciones de procedimiento se producían en la mayor parte de las sesiones de resolución de problemas codificadas (un margen del 7 I-100 por $100 \mathrm{del}$ tiempo disponible). Se definieron como todas las actividades realizadas por uno o ambos niños que se concentraban en la realización de la tarea ${ }^{3}$. Algunos ejemplos de interacciones de procedimiento son la distribución y ordenación de los materiales, la elección de los productos químicos, anotar los experimentos, etcétera. Se identificaron tres niveles de interacciones de procedimiento: paralelo, asociativo, cooperativo (adaptados de los estudios de Parten sobre la interacción social, i932). Estos tres niveles representan tres enfoques cualitativamente diferentes del fenómeno de compartir ideas y de la división del trabajo. Durante las interacciones de procedimiento paralelas, los niños comparten materiales e intercambian comentarios acerca de la tarea. Sin embargo, llevan a cabo pocos $\longrightarrow$ ninguno- intentos de controlar el trabajo del otro o de informar ai otro de sus propios pensamientos y acciones. Las interacciones de procelimiento asociativas se producen cuando los niños intentan intercambiar información acerca de las combinaciones que cada uno ha seleccionado. Sin embargo, en el nivel asociativo, no se lleva a cabo ningún intento de coordinar los roles de los dos compañeros. Las interacciones de cooperación exigen que ambos niños controles el trabajo del otro, jugando papeles coordinados en la realización de los procedimientos de trabajo.

El código de las estrategias de experimentación se adaptó de Kuhn y Phelps (en prensa). Se observaron tres tipos básicos de estrategias: una estrategia de azar o de ensayo y error; una estrategia de aislamiento de variables, y una estrategia combinatoria. La estrategia de experimentos al azar representa un enfoque de la experimentación poco sistemático y relativamente ineficaz. La estrategia de aislamiento de variables es efectiva sólo para la resolución de los tres primeros problemas. Los problemas más avanzados, del cuarto hasta el séptimo, exige tanto el aislamiento experimental de las variables como las estrategias combinatorias. Este sistema de codificación tiene como fin identificar cuándo se produce un cambio de estrategia (desde el aislamiento de una sola variable al aislamiento de ambas variables y la combinatoria), si es que se produce.

El código de estrategias experimentales se aplicó teniendo en cuenta únicamente el tipo de experimentos químicos orga- 
nizados. Ni el tipo de organización social utilizado para seleccionarlos ni los tipos de conversación observada durante los procesos de organización afectaron a la aplicación del código. Así, la codificación de las estrategias experimentales constituia una valoración del comportamiento de cada pareja independiente de la obtenida al codificar sus interacciones sociales.

Para la comparación de los logros en la solución de problemas obtenidos por los sujetos que trabajaban en régimen de colaboración y los que lo hacian en régimen individual, se disponia de dos datos: el número de problemas químicos resueltos durante las once sesiones y la diferencia de puntualización entre el pretest y el postest. La primera comparación muestra unas diferencias sorprendentes entre la colaboración y la resolución individual de problemas. Mientras Kuhn y Ho observaron que sólo cuatro de los is niños trabajando en régimen individual habian resuelto los problemas del 1 al 3 en las once sesiones, en el mismo periodo de tiempo las cuatro parejas de Forman resolvieron los problemas del 1 al 4. Además, una pareja (George y Bruce) resolvió los problemas del 1 al 6 durante este período de tres meses, un logro al que no se habia acercado ninguno de los sujetos de Kuhn y Ho.

Las comparaciones entre el pretest y el postest de los sujetos que trabajaron en régimen individual $y$ de las parejas produjeron unos resultados más mezclados. Estos resultados aparecen en las tablas I y 2 (ignoremos por ahora las iniciales entre paréntesis). En el «simple plant problem" (cuadro I), los sujetos trabajando en régimen individual mostraron mayor progreso que las parejas entre el pretest $y$ el postest. Contrastando con esto, los sujetos que habian trabajado por parejas parecian mostrar un mayor progreso en el problema de las combinaciones (cuadro 2) que los sujetos que trabajaron individualmente ${ }^{4}$. Así, mientras las

CuADro I

Frecuencias de las categorias de respuestas del pretest y postest en el problema de la planta simple (simple plant problem)

\begin{tabular}{|c|c|c|c|c|}
\hline \multirow[b]{2}{*}{ Grupo } & \multicolumn{4}{|c|}{ Puntuacióń del pretest } \\
\hline & $\begin{array}{l}\text { Predominant. } \\
\text { concreto }\end{array}$ & Transicional & $\begin{array}{l}\text { Predominant. } \\
\text { formal }\end{array}$ & Total \\
\hline $\begin{array}{l}\text { Individual } \ldots \ldots \\
\text { Parejas } \ldots \ldots\end{array}$ & $\begin{aligned} 15 \\
8\end{aligned}$ & $\begin{array}{l}\circ \\
\circ\end{array}$ & $\begin{array}{l}\circ \\
\circ\end{array}$ & $\begin{aligned} 19 \\
8\end{aligned}$ \\
\hline & \multicolumn{4}{|c|}{ Puntuaciones del postest } \\
\hline Grupo & $\begin{array}{l}\text { Predominant. } \\
\text { concreto }\end{array}$ & Transicional & $\begin{array}{l}\text { Predominant. } \\
\text { formal }\end{array}$ & Total \\
\hline $\begin{array}{l}\text { Individual } \\
\text { Parejas ... }\end{array}$ & ${ }_{6}^{4}\left(\mathrm{Ml}, \mathrm{L}_{2}, \mathrm{M}_{2}, \mathrm{G}, \mathrm{K}_{3}, \mathrm{~K}_{2}\right)$ & $\stackrel{5}{(B)}$ & $\begin{array}{c}6 \\
\mathrm{I}\left(\mathrm{L}_{1}\right)\end{array}$ & $\begin{array}{r}15 \\
8\end{array}$ \\
\hline
\end{tabular}

CUADRO 2

Frecuencias de las categorias de respuestas del pretest y postest en el prblema de combinaciones

\begin{tabular}{|c|c|c|c|c|}
\hline \multirow[b]{2}{*}{ Grupo } & \multicolumn{4}{|c|}{ Puntuación del pretest } \\
\hline & $\begin{array}{l}\text { Predominant. } \\
\text { concreto }\end{array}$ & Transicional & $\begin{array}{c}\text { Predominant. } \\
\text { formal }\end{array}$ & Total \\
\hline $\begin{array}{l}\text { Individual } \ldots . \\
\text { Parejas } \ldots . . .\end{array}$ & $\begin{array}{r}15 \\
8\end{array}$ & $\begin{array}{l}\circ \\
\circ\end{array}$ & $\begin{array}{l}\circ \\
\circ\end{array}$ & $\begin{array}{r}15 \\
8\end{array}$ \\
\hline & \multicolumn{4}{|c|}{ Puntuación del postest 1} \\
\hline Grupo & $\begin{array}{l}\text { Predominant. } \\
\text { concreto }\end{array}$ & Transicional & $\begin{array}{c}\text { Predominant. } \\
\text { formal }\end{array}$ & Total \\
\hline $\begin{array}{l}\text { Individual } \ldots . \\
\text { Parejas } \ldots \ldots\end{array}$ & $s_{12}^{12}\left(K_{2}, L_{1}, M_{1}, K_{1}, G\right)$ & $3\left(\mathrm{~L}_{2}, 3^{\mathrm{M}} \mathrm{B}, \mathrm{B}\right)$ & $\begin{array}{l}\circ \\
\circ\end{array}$ & $\begin{array}{r}15 \\
8\end{array}$ \\
\hline
\end{tabular}


parejas parecian dominar las series de problemas químicos a una velocidad mucho mayor que los que trabajaban individualmente, no mostraron unos adelantosconsistentemente mayores en la relación pretest-postest.

Una clara diferencia entre estas dos comparaciones (el progreso a través de los problemas versus cjecución en el postest) es que ambos compañeros fucron capaces de contribuir a la solución de cada uno de los problemas químicos presentados, pero en las mediciones pretestpostest los compañeros estaban solos. Las estrategias de solución de problemas relativamente sofisticadas que los colaboradores podian llegar a mostrar cuando podian ayudarse mutuamente no eran tan aparentes cuando cada compañero tenia que trabajar sólo en problemas similares.

Otra razón que justifica por qué los colaboradores no siempre superaban en resultados a los que trabajaban individualmente puede que radique en las diferencias entre los compañeros. Debido a la pequeña cantidad de parejas examinadas, las grandes diferencias entre parejas pueden oscurecer todos los aspectos excepto las masivas diferencias entre parejas $y$ sujetos trabajando en régimen individual.

Volvamos, por tanto, al segundo grupo de comparaciones: las efectuadas entre las parejas. En primer lugar, vamos a examinar los tipos de interacciones sociales producidas durante ese tiempo en las tres parejas que trabajaban en régimen dé colaboración. En segundo lugar, observaremos las estrategias de experimentación utilizadas por esas mismas parejas. En tercer lugar, examinaremos los resultados pretest-postest.

La diferencia más obvia entre los comportamientos sociales de las tres parejas concierne a la evolución de las interacciones de procedimiento. Todas las interacciones de procedimiento se clasificaban como paralelas, asociativas o cooperativas. El cuadro 3 muestra que las tres parejas adoptaron interacciones predominantemente paralelas y asociativas durante la primera sesión codificada (la sesión tres para todas las tres parejas). Sólo Lisa y Linda mostraton un cierto grado de comportamiento cooperativo durante esta sesión. Sin embargo, en las sesiones, 8 y i I, George y Bruce eran totalmente cooperativos. I.isa y linda conservaban ciertos patrones de interacción asociativa aun en la sesión $s$, pero en las 9 y 11 también adoptaron interacciones cooperativas. Contrastando con ello, Matt y Mitch no cooperaron nunca a lo largo del periodo de tres meses. El modelo de interacción que Matt y Mitch parecian preferir era de naturaleza predominante o enteramente paralela.

CUADRO 3

Porcentaje de tiempo dedicado a actividades paralelas, asociativas o cooperativas

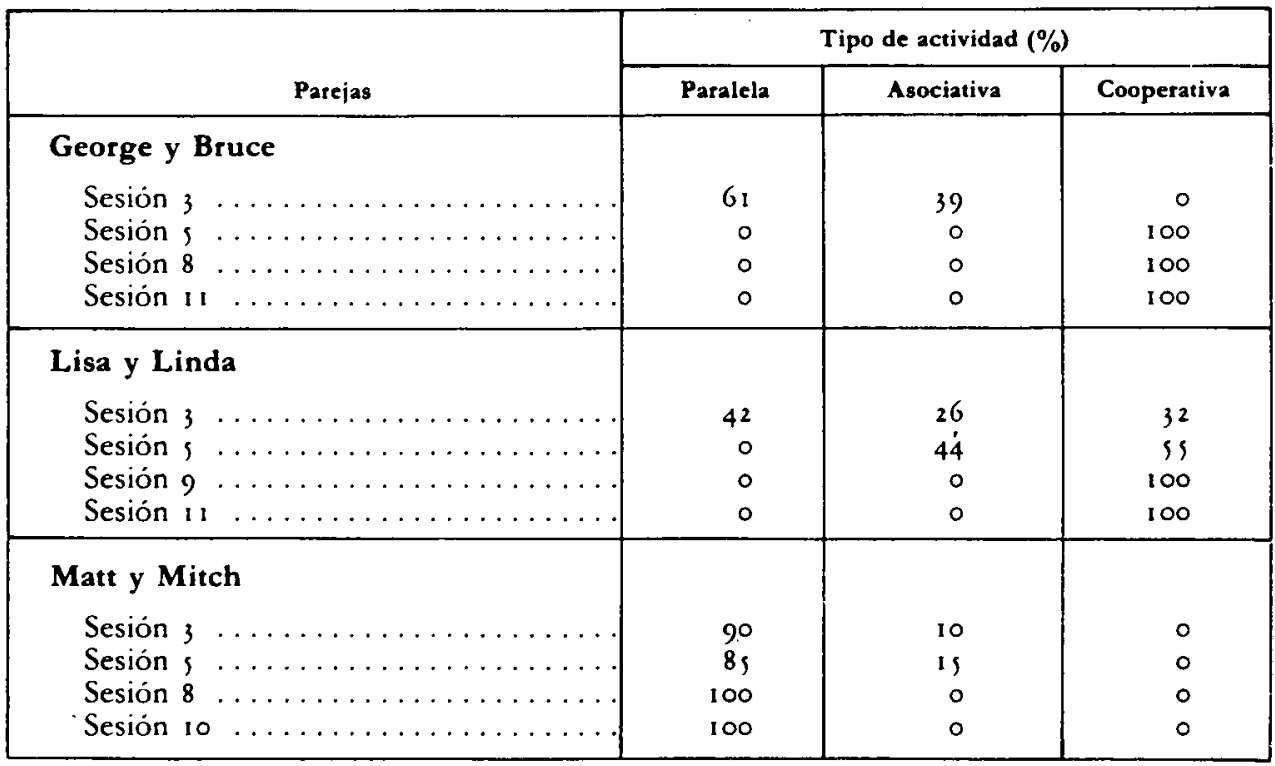


El cuadro 4 resume las diferencias en estrategias de experimentación utilizadas en las dos últimas sesiones por cada pareja. Las tres parejas utilizaron tipos de estrategias de experimentación similares durante las sesiones anteriores. George y Bruce, la pareja que resolvió el mayor número de problemas, en las dos últimas sesiones utilizó tanto una estrategia de aislamiento de variables como combinatoria. Lisa y Linda utilizaron sólo la estrategia de aislamiento de variables en la sesión 9, pero ambas estrategias en la sesión I I. Contrastando con ello, Matt y Mitch produjeron, bien experimentos al azar, bien experimentos capaces de aislar variables simples a lo largo de todo el estudio, a pesar del hecho de que ninguna de estas estrategias fue suficiente para resolver los avanzados problemas a los cuales se les sometió durante las sesiones $8 \mathrm{y}$ io.

\section{CuAdro 4}

Estrategias de experimentación empleadas en los problemas quimicos 4-7

\begin{tabular}{|c|c|c|c|}
\hline \multirow{2}{*}{ Parejas } & \multicolumn{3}{|c|}{ Estrategia de experimentación } \\
\cline { 2 - 4 } & $\begin{array}{c}\text { Combinaciones } \\
\text { al azar }\end{array}$ & $\begin{array}{c}\text { Estrategia de } \\
\text { 2islamiento de } \\
\text { variables }\end{array}$ & $\begin{array}{c}\text { Estrategia } \\
\text { combinatoria } \\
\text { sistemática }\end{array}$ \\
\hline $\begin{array}{c}\text { George y Bruce } \\
\text { Sesión 8 } \\
\text { Sesión I }\end{array}$ & & $\mathrm{X}$ & \\
\hline Lisa y Linda & & $\mathrm{X}$ & $\mathrm{X}$ \\
Sesión 8 & & $\mathrm{X}$ & \\
Sesión I & & $\mathrm{X}$ & $\mathrm{X}$ \\
\hline Matt y Mitch & & & \\
Sesión 8 & & $\mathrm{X}$ & \\
Sesión 10 & $\mathrm{X}$ & $\mathrm{X}$ & \\
\hline
\end{tabular}

Volviendo a las mediciones pretes-postest, encontramos que George y Bruce, que habian trabajado tan bien juntos, no mantuvieron este elevado nivel de realización al ser examinados individualmerıte. Las iniciales que aparecen en los cuadros 1 y 2 muestran el nivel posterior de los seis niños cuyas cintas fueron analizadas: George $(G)$; Bruce $(B)$; Lisa (LI); Linda (L2); Matt (M 1 ); Mitch (M2); más la cuarta pareja no analizada ( $\mathrm{K}_{\mathrm{I}}$ y $\mathrm{K}_{2}$ ). En el problema de planta simple (cuadro I), los niños que recibieron la puntuación más elevada fueron Bruce y Lisa; en el problema de combinación (cuadro 2), Bruce, Linda y Mitchell exhibieron el nivel de razonamiento más avanzado. Asi, las claras diferencias entre parejas, aparentes en los vídeos de las sesiones de solución de problemas en régimen de colaboración, no se reflejaron en los resultados del postest.

En resumen, cuando se comparan las parejas con los sujetos trabajando individualmente, las parejas resuelven los pro- blemas de combinación química a una velocidad mucho mayor. Sin embargo, las parejas no son mejores que los que han trabajado en régimen individual en todas las mediciones del postest. Estos últimos parecen superar a las parejas en el "plant simple problem», test de habilidad del sujeto para aislar variables, mientras que las parejas parecen ser mejores en los problemas de combinación.

Cuando se hacen comparaciones entre las parejas, se ve que George y Bruce resuelven más problemas de combinación química que las otras parejas. Además, George y Bruce fueron los primeros en desplazarse hacia un modelo de interacción completamente cooperativo, y en utilizar una estrategia de experimentación combinatoria. En alguna de estas variables, por ejemplo, el grado de cooperación demostrado y el uso de una estrategia combinatoria, Lisa y Linda ocuparon una posición intermedia entre los dos pares de chicos. Sin embargo, estas considerables diferencias en el exilio de inte- 
racción adoptado y en la estrategia empleada para la resolución de los problemas no se refleja en las realizaciones posteriores de estos niños. En general, George y Bruce no mostraron en el postest niveles de razonamiento consistentemente superiores a los de los otros sujetos.

\section{DISCUSION}

¿Qué nos pueden decir estos resultados acerca de la hipótesis propuesta por Perret-Clermont según la cual la interacción entre compañeros puede inducir un conflicto congnitivo que, a su vez, desemboca en una reestructuración cognitiva y en el progreso intelectual? Forman encuentra una asociación entre los niveles superiores de coordinación social (interacciones cooperativas de procedimiento) y el uso de ciertas estrategias de experimentación (estrategias combinatorias). Sin embargo, no planeó una medida de conflicto cognitivo en su estudio, y sus hallazgos no pueden, por tanto, establecer que la coordinación, a su vez, afecta a la capacidad de resolución de problemas.

Una razón por la cual no se pudo evaluar el conflicto cognitivo era que los indicios claros de conflicto, por ejemplo, las discusiones, eran relativamente escasos durante la parte de las sesiones de resolución de problemas examinadas, la fase de planificación de la tarea durante la cual eran más aparentes las estrategias de experimentación.

En esta parte de la sesión los sujetos podian hacer hipótesis relativas a los experimentos, pero no podian verificarlos. Durante la mayor parte del tiempo de planificación los niños estaban ajetreados trabajando por separado o conjuntamente, ordenando y compartiendo los materiales de trabajo y planeando y eligiendo los experimentos. Entre los niños que interactuaban en un nivel cooperativo se intercambió una gran cantidad de ayuda, ánimos, corrección de errores e ideas. Por ejemplo, un niño seleccionaba las combinaciones químicas mientras el otro verificaba los duplicados. En lugar de puntos de vista en conflicto se veia a dos personas intentado construir y llevar a cabo un plan de experimentación conjunto para más tarde verificarlo.
Los puntos de vista en conflicto aparecían después en las sesiones de solución de problemas, cuando la mayor parte o todos los resultados de los experimentos eran visibles. En ese momento se podia observar a los niños formando conclusiones distintas y a veces opuestas sobre la solución del problema. Un conflicto de este tipo se presentó en la sesión de solución de problemas \# 3 entre George y Bruce: a continuación presentamos un resumen de su interacción tomado del material grabado en video.

En esta sesión, sólo el producto quimico $C$ era la solución al problema químico. Los dos chicos organizaron y mezclaron el siguiente conjunto de experimentos: $B, C, B E, C D, C E, D E$, BDC, CDF, DEF. Además, pudieron examinar los resultados de los dos experimentos de demostración: $\mathrm{BCE}, \mathrm{DEF}$. Todos los experimentos que contenian el producto quimico $C$ se volvian de color morado, mientras que el resto permanecia transparente.

Tras llevar a cabo todas las combinaciones previstas, el experimentador preguntó a los niños, «¿qué creéis que hace que se vuelva morado?" En un principio, Bruce concluyó que la respuesta era $C$ y E. George manifestó su sorpresa de que un solo elemento, por ejemplo, el C, pudiese producir el cambio de color deseado. En respuesta a la observación estándar del experimentador, "¿estás seguro de que es C y E?", Bruce reexaminó algunos experimentos $y$ encontró uno que contenía $\mathrm{E}$ ( $\mathrm{y}$ no $\mathrm{C}$ ) que no habia cambiado de color. Sin embargo, Bruce no llegó a la conclusión de que $C$ era el único producto quimico operativo. Entonces, George preguntó a Bruce si todos los experimentos que contenian $C$ producian el cambio de color deseado. Bruce examinó cuidadosamente todos los experimentos conteniendo $C$ y anunció que todos ellos cambiaban el color.

Basándose en la evidencia experimental y en alguna información que recordó de sesiones anteriores, George llegó a la conclusión de que $C$ era la solución al problema. Bruce, sin embargo, contradijo a George afirmando que era $F$. En ese momento, ambos reexaminaron los experimentos. Al finalizar, George seguia concluyendo que era $C$ y Bruce que era $C$ y $F$.

El experimentador les preguntó si podian estar seguros de sus respuestas. George respondió que estaba seguro de 
$C$ pero no de $F$. De nuevo se volvió a examinar la evidencia. Esta vez, Bruce identificó el experimento CDF como indicador de que $F$ era un producto quimico operativo. George rebatió este argumento comparándolo con el experimento DEF que no producia la reacción deseada. Bruce respondió que $\mathrm{D}$ ! $E$ eran liquidos más fuertes que $F y$ por eso impedian que $F$ actuase. Entonces George intentó otro camino preguntando a Bruce cómo podia saber que era $F$ y no $C$ el que hacía que la mezcla CDF se volviese morada. Bruce replicó preguntando a George cómo podia asegutar que no eran tanto $C$ como $F$ los que hacian que CDF se volviese morada. La respuesta concluyente de George fue una afirmación de que, simplemente, sabia que era $C$ solo.

Este intercambio muestra el tipo de actividades que parecen inducir las soluciones conflictivas al problema. I os niños volvieron repetidamente a la evidencia experimental en busca de datos que les sirviesen de justificación. Puesto que las conclusiones a las que habian llegado eran diferentes, se veían obligados a admitir información que contradecía sus propias deducciones, asi como los datos que las justificaba. Después, estos datos debian ser integrados en el seno de un argumento convincente para justificar su punto de vista. También debian construir contraargumentos a la posición adoptada por el compañero. Bruce, en particular, se vio obligado a revisar sus conclusiones basándose en la evidencia que George le señaló. A pesar de sus esfuerzos, George fue incapaz de convencer a Bruce de que aceptase sus conclusiones. Desgraciadamente, no habian acumulado una cantidad suficiente de evidencia experimental adecuada en la sesión \# 3 que les permitiese alcanzar un consenso acerca de la solución correcta.

Así, la colaboración en la tarea de combinación química parece que abarca dos tipos diferentes de procesos sociales interactivos. El primer proceso, que tiene lugar durante el estadio de organización o planificación de la tarea, incluye tanto patrones que funcionan por separado (paralelos) como patrones cooperativos estrechamente coordinados. La cooperación a lo largo del estadio de organización consiste en la guia, ánimos y ayuda mutuas. A menudo, durante esta fase del trabajo se asumen roles complementarios
Más adelante, cuando se examina la evidencia experimental, aparece el segundo tipo de proceso interactivo. En ese momento parece como si cada niño llegara a conclusiones independientes sobre la solución de la tarea, basadas en toda o sólo una parte de la evidencia experimental de la cual dispone. Una vez que cada niño ha llegado a una conclusión puede encontrarse con que su compañero o compañera no está de acuerdo. En esta circunstancia se plantean claras perspectivas de conflicto sobre la evidencia experimental que se manifiestan bajo la forma de discusión abierta. Los argumentos capaces de producir el consenso parecían ser los que utilizaban correctamente la evidencia que los justificaba.

Parece que donde mejor se puede probar la idea de Perret-Clermont de que el conflicto cognitivo es el mediador entre la interacción entre compañeros y la reorganización cognitiva, es en contextos en los que se supone que van a producirse manifestaciones abiertas de conflicto. Estos contextos se producen cuando los niños disponen de una considerable cantidad de evidencia empírica, cuando esta evidencia es capaz de sugerir por lo menos dos soluciones distintas al problema, y cuando se exige una solución de consenso.

La hipótesis de Perret-Clermont sobre la importancia del conflicto cognitivo proviene de la teoria de Piaget relativa al papel de los factores sociales en el desarrollo. La mayor parte de las investigaciones realizadas sobre la colaboración entre iguales se ha basado en las ideas de Piaget. Piaget daba más importancia a la interacción entre iguales que a la interacción adulto-niño, de modo que no es extraño que la mayor parte de las investigaciones sobre colaboración hayan compartido la perspectiva piagetiana.

Para comprender tanto los puntos fuertes como los débiles de esta perspectiva sobre la colaboración, es necesario apreciar el rol que juega la interacción entre compañeros en la teoria de Piaget. Piaget (1930) ha identificado cuatro factores que considera necesarios para una teoría del desarrollo cognitivo: la maduración, la experiencia con el medio físico, las experiencias sociales y la equilibración o autoregulación. Además, Piaget afirma que la equilibración es el factor más fundamental de los cuatro. La interacción entre 
compañeros, y las experiencias sociales en general, derivan su importancia de la influencia que pueden ejercer sobre la equilibración a través de la introducción del conflicto cognitivo. Perret-Clermont comparte este punto de vista cuando escribe:
1
"Listá claro que un conflicto cogniti- vo de este tipo no crea las formas de las operaciones, pero produce el desequili- brio que hace necesaria la elaboración cognitiva, y de este modo el conflicto cognitivo confiere un papel especial al factor social, como uno de los varios factores que dan lugar al crecimiento mental. El conflicto sociocognitivo pue- de verse como similar al papel que juega un catalizador en una reacción química: no aparece para nada en el producto final, pero, sin embargo, es indispensable para que se produzca !a reacción." (Perret-Clermont, 1980, pági- na 178 .)

Cuando Piaget se fija en la interacción entre compañeros, por tanto, busca la evidencia del desequilibrio, es decir, del conflicto cognitivo. No le interesa describir o explicar los procesos sociales interactivos como un todo. La teoría de Piaget es de gran ayuda para explicar aquellas situaciones en las cuales el conflicto cognitivo se expresa de forma clara y abierta en comportamientos sociales externos, como, por ejemplo, las discusiones. En cambio, es de menor ayuda para entender aquellas situaciones en las cuales el conflicto abierto no es aparente, pero donde están presentes la dirección y ayuda mutuas. Afortunadamente, los escritos de Vygotski sobre la interacción adultoniño subrayan el valor intelectual de este tipo de interacción entre compañeros.

Para ilustrar cómo las ideas de Vygotski siempre aportan claridad sobre algunos de los procesos que forman parte de la colaboración entre iguales, discutiremos otro conjunto de observaciones de George y Bruce. Uno de los descubrimientos más sorprendentes del estudio de Forman es la discrepancia que hay en la manera de funcionar de una diada como unidad y la manera de funcionar sus componentes por separado. Sin duda alguna, George y Bruce eran los colaboradores con más éxito $y$, sin embargo, no mostraron el mismo alto nivel de funcionamiento cuando realizaron el postest por separado. Esta discrepancia entre niveles de realización individuales y por parejas resultaba también aparente cuando los sujetos que trabajaban en régimen de colaboración eran comparados con quienes trabajaban en régimen individual. En las mediciones del postest, administradas individualmente, quienes habian trabajado en régimen de colaboración no obtuvieron mejores resultados que quienes habían trabajado en solitario. Sin embargo, los compañeros que trabajaban juntos fueron capaces de resolver muchos más problemas quimicos, en el mismo período de tiempo, que los que habian trabajado individualmente.

Vygotski aceptaba que pudiese existir una discrepancia entre la resolución individual y social de problemas cuando desarrolló su concepto de zona de desarrollo próximo. La definió como «la distancia entre el nivel de desarrollo real, determinado por la solución individual de problemas, y el nivel de desarrollo potencial, determinado a través de la solución de problemas bajo la dirección de un adulto o en colaboración con compañeros más capaces» (1978, pág. 86). Así, Vygotski formulaba la hipótesis de que los niños serían capaces de resolver problemas con la ayuda de un adulto o de compañeros más capaces antes de que los pudiesen resolver solos. Esta observación, aparentemente obvia, fue utilizada para extraer varias conclusiones originales. Una conclusión fue que la zona de desarrollo próximo podía ser utilizada para identificar aquellas habilidades más predispuestas para la instrucción. Otra fue que el aprendizaje consiste en la interiorización de procesos sociales interactivos. Según Vygotski, el desarrollo tiene lugar cuando la regulación interpsicológica se transforma en regulación intrapsicológica.

Volviendo a los datos de Forman parece que se puede producir un proceso similar de regulación interpsicológica a intrapsicológica en contextos cooperativos en los que ninguno de los compañeros puede verse como objetivamente «más capaz», pero en los que los compañeos pueden asumir roles sociales separados, aunque complementarios. Un niño puede adoptar un rol de observación, de guía y corrección, mientras el otro realiza la tarea propiamente dicha. Parece que este compañero que observa suministra un tipo de ayuda como la que Wood, Bruner y Ross denominan «de andamiaje» (1976). 
La ayuda que proporciona un compañero observador parece permitir a los dos colaboradores la resolución conjunta de problemas antes de que sean capaces de resolver el mismo problema por separado. Cuando los colaboradores asumen los roles complementarios comienzan a parecerse a los compañeos tutores de los cuales hemos hablado antes. Por ejemplo, los roles de observador/realizador son funcionalmente similares a los de crítico/autor observados en la clase de New Hampshire de Egan.

Además, a partir de los datos de Forman, se pueden ver casos en que los que las estrategias de solución de problemas aparecen primero como procedimientos interactivos sociales y que luego se interiorizan. Recordemos que se administró un problema combinatorio a cada niño individualmente en tres momentos diferentes (como pretest, como postest inmediato y como postest más alejado en el tiempo). Además, estos mismos niños se enfrentaban a un problema combinatorio similar en cada sesión de solución de problemas, cuando se les pedía que, juntos, decidiesen qué mezclas químicas preparar. Por tanto, es posible realizar una comparación entre las combinaciones generadas por cada niño trabajando individualmente o por parejas.

Tanto Georges como Bruce emplearon una estrategia empirica para generar combinaciones durante el pretest. Por ejemplo, seleccionando una combinación al azar $y$ luego basando la siguiente combinación en la primera, añadiendo, eliminando o sustituyendo uno de sus elementos. La tercera combinación la producirian copiando, con alguna revisión de menor importancia, la segunda combinación. La comprobación por parejas de cada nueva combinación con cada combinación previa era el procedimiento empírico utilizado para prevenir las repeticiones.

En sus primeras sesiones de resolución de problemas en régimen de cooperación, George y Bruce trabajaron en paralelo y cada uno utilizaba una estrategia empírica similar a la utilizada en el pretest para generar combinaciones. Tras un mes de trabajar juntos idearon un procedimiento social para generar combinaciones empíricamente, asumiendo roles complementarios para la solución de problemas: uno seleccionaba los productos químicos y el otro verificaba su singularidad.
Al cabo de dos meses habian empezado a organizar sus combinaciones en grupos según el númeo de elementos. Además, habian ideado un sistema deductivo para generar combiaciones de dos elementos. Este procedimiento deductivo permitia al niño que habia establecido previamente las verificaciones, proponer, corregir y reforzar las selecciones de su compañero. Empíricamente se produjeron combinaciones de un orden superior al utilizar el procedimiento social familiar.

En la última sesión los niños siguieren asumiendo roles complementarios, pero entonces utilizaron la pizarra como instrumento de registro. Produjeron combinaciones de una forma aitamente organizada - combinaciones a simples de dos elementos, de tres, etc.- y fueron capaces de generar la casi totalidad de las 3 I combinaciones posibles. Utilizaron un procedimiento deductivo para generar las combinaciones de dos elementos, pero siguieron basándose en su procedimiento empírico para las combinaciones de un orden superior.

En el primer postest, realizado al zabo de una semana de haber finalizado la última sesión de colaboración, se verificó el grado de interiorización del sistema combinatorio deductivo de cada niño pidiéndoles que generasen combinaciones independientemente. Bruce fue capaz de generar por si solo, deductivamente, las diez combinaciones de dos elementos, pero George no. George utilizó un sistema empírico para generarlas. Sin embargo, en el segundo postest, realizado cuatro meses más tarde, ambos niños habian interiorizado un procedimiento deductivo para producir combinaciones de dos elementos.

Parece que estos dos niños fueron capaces de aplicar una regla intrapsicológica preexistente, un procedimiento combinatorio empírico, en un contexto cooperativo dividiendo el procedimiento en roles complementarios. Con una exposición repetida al problema, ambos niños fueron capaces de progresar hasta un procedimiento deductivo para generar combinaciones simples, de dos elementos. Al principio, el razonamiento deductivo era claramente una actividad social, tanto para George como para Bruce. Cada vez que uno seleccionaba una serie de combinaciones, el otro le guiaba, proponía y corregía en sus selecciones. Más tarde, uno de los compañeros pudo demostrar que había interiorizado este procedimien- 
to deductivo utilizándolo para generar todas las combinaciones posibles de dos elementos, trabajando individualmente. Cuatro meses más tarde, ambos compañeros fueron capaces de generar, por su cuenta, todos los pares posibles de cinco objetos utilizando un método deductivo. Así, para estos dos niños, el razonamiento combinatorio deductivo apareció, en primer lugar, en un contexto cooperativo. Sólo uno de los dos niños fue capaz, inicialmente, de mostrar que habia interiorizado este procedimiento cuando generó combinaciones solo. Sin embargo, meses más tarde, ambos niños habian interiorizado este proceso deductivo.

En resumen, una perspectiva piagetiana sobre el papel de los factores sociales en el desarrollo puede resultar útil para comprender aquellas situaciones en las cuales se hallan presentes claros indicios de conflicto cognitivo. Sin embargo, si se quiere comprender las consecuencias cognitivas de otros contextos interactivos sociales, las ideas de Vygotski parecen más aplicables. En las tareas en las que se generaba una evidencia experimental y en las que se necesitaban habilidades de dirección al asumir roles complementarios en la resolución de problemas, los compañeros podian realizar tareas juntos antes de que fuesen capaces de realizarlas individualmente. Parece como si el encargado de observar suministrase algo asi como una ayuda de "andamiaje", similar a la que autores han atribuido al adulto en contextos de enseñanza.

Asi, la perspectiva vygotskiana nos permite ver que las tareas cooperativas que requiren generación de datos, planificación y dirección pueden suponer otro conjunto de valiosas experiencias para los niños. En las tareas de este tipo se necesita construir un bloque común de suposiciones, procedimientos e información. Estas tareas exigen a los niños que integren sus diferentes concepciones del trabajo para elaborar un plan mutuo. Una forma de lograr una perspectiva común del trabajo es asumir roles complementarios en la resolución de problemas. Cada niño aprende a utilizar el lenguaje para guiar las acciones de su compañero y, a su vez, a ser guiado por el lenguaje del compañero. La exposición a esta forma de regulación social puede permitir a los niños el dominio de problemas dificiles antes de que sean capaces de resolverlos individualmente. $Y$, lo que es más importante, la experiencia con formas sociales de regulación puede suministrar a los niños las herramientas necesarias para dominar por ellos mismos dichos problemas. Les permite observar y captar el proceso de resolución de problemas como una totalidad y seleccionar aquellos procedimienos que resultan más eficaces. Cuando pueden aplicar esta comprensión social a si mismos están en situación de resolver, independientemente, aquellas tareas que antes habían sido capaces de resolver con ayuda.

Este conjunto de tareas parece suministrar algunas de las experiencias que les provee la tutoria entre compañeros: la necesidad de dar instrucciones verbales a los compañeros; la necesidad de autorreflexión que puede suponer un público visible; la necesidad de responder a preguntas y retos del compañero. El modelo recíproco de ayuda entre compañeros que sirve para caracterizar a los niños en la clase de Egan es incluso más aparente en contextos colaborativos de resolución de problemas similares a los observados por Forman.

\section{CONCLUSION}

En estos análisis no estamos hablando de una cultura infantil separada de los adultos. Lo que I.eontiev y Luria presentan como la «forma más importante especificamente humana de desarrollo mental" - es decir, "la asimilación de la experiencia humana general en el proceso de enseñanza»- se debe fundamentar, en última instancia, en las interacciones adulto-niño. Pero las relaciones entre compañeros pueden funcionar como contextos de transformación intermedios entre las interacciones sociales $y$ externas adultoniño y el lenguaje interior individual del niño.

Sil bien este tipo de interacciones entre compañeros tienen lugar en casa y en la comunidad tanto como en la escuela, pueden ser especialmente importantes en esta última, debido a las limitaciones y rigideces caracteristicas de las interacciones adulto-niño en tales marcos institucionales. Cazden (1979) subraya el valor que tienen para el niño las interacciones padres-niño, tales como el juego del cu-cú o la lectura conjunta de libros de cuentos. 
En interacciones de este tipo hay una estructura predecible en la cual en un principio el adulto lleva todo el peso de la actuación $y$, lentamente, el niño va adoptando un papel cada vez más actiro, llegando a hacer todos los papeles que inicialmente hacia la madre y el padre. El contraste entre tales medios de aprendizaje $y$ el aula es impresionante. En las lecciones escolares, los profesores dan órdenes y los niños las llevan a cabo en silencio; los profesores hacen preguntas y los niños las responden, a menudo con una sola palabra o frase. $Y$, lo que es más importante, estos roles no son reversibles, por lo menos no dentro del contexto de las interacciones profesor-alumno. L.os niños no dan nunca órdenes al profesor ! escasean las preguntas dirigidas al profesor, excepto con el fin de pedirle permiso para algo. El único contexto en el que los niños pueden invertir los roles interactivos con el mismo contenido intclecrual, dando órdenes, así como siguiéndolas, haciendo preguntas $y$ contestándolas, es con sus compañeros.

\section{Notas}

1 I.a investigación de Forman fue subvencionada en parte por una beca del Radclifte College, una ayuda a Deanna Kutn de la Fundación Milton, Liniversidac de iialiard, y por una beca NilMll número $T_{32}$ MIII 786 al departamento de psicologia de la universidad de Northwestern. Quisiéramos dar las gracias a los estudiantes, a la Faculad $y$ al director de la Stratoon Filementary School, Arlinton, Massachusetts, por su generosa participación en esta investigación; $y$ a leonard Scinto, Addison Stone Jim Wertsh por sus valiosos comentarios a anteriores esbozos de este capitulo.

2 Es importante subrayar aqui el trabajo pionero de Brian Simon, de la Lniversidad de Leicester, Inglaterra, al editar traducciones de las obras soviéticas sobre psicologia y psicología educativa para los hablantes ingleses (B. Simon, 1997; B. Simon \& Simon, 1963). Es también el segundo autor del estudio observacional de la educación primaria en Inglaterra antes citado (Gaton, Simon y Croll, i980).

3 Se utilizaron otros códigos de interacción social para identificar conversaciones que servían para planificar, reflexionar sobre u organizar estos procedimientos (interacciones de metaprocedimientus); bromas centradas en el trabajo (interacciones lúdicas); observaciones centradas en el trabajo (observaciones compartidas) y comportamiento desconectado del trabajo.

4 Se administró un segundo conjunto de postest a ambas muestras al cabo de cuatro meses del primer postest. De forma constante, las parejas superaron a los niños que habian trabajado en régimen individual en las dos mediciones de este postest. Sin embargo, la interpretación de estos resultados es problemática, debido a que este periodo de cuatro meses coincidia con el curso escolar en el caso de las parejas, pero con el verano para los niños que habian trabajado individualmente.

\section{Referencias}

CARRASCO, R. I.; VFRA, A., y CAJDFin, C. B.: "Aspects of bilingual students, communicative competence in the classroom: a case study". Fn R. Di R.i. (F.): Latino language and communicative behavior. Discourse processes: Adrances in research and theory, vol: 4. Norwood, N. J.: Ablex Pub., en prensa.

CiZIDIN, C. B.: "Peekaboo as an instructional model: discurse development at school and at home». Fn: Papers and Reports on Child Language Detelopment. Núm. 17. Standford Liniversity: Dept. of linguistics 1979, 1-29. Versión revisada en: $\mathrm{B}$. Bain (Ed.), The sociogenesis of language and buman conduct: A multi-disciplinary book of readings. N. Y.: Plenum, en prensa.

CALDEN, C. B. et al.: "You all gonna bafta listen: peer teacbing in a primary classroom». In W". A. Collins (Ed.), Children's language and communication. izth Annual Minnesota Symposium on Child Development. Hillsdale, N. J. Lawrence Erlbaum, 1978, $183-231$.

Doise, W: MLGivi, G., y PERRET.Ci.ermont, A. N.: "Social interaction and the development of cognitive operationsm. Huropean Journal of Social Psycbology, 1975, , (3), 367-383.

Doisf, W.; Migivi, G., y Perret.Ciffriont, A. N.: "Social interaction and cognitive development: Further evidence». European Journal of Social Psycbology, 1976, 6, 245-247.

Formin, F.. A.: The role of collaboration in problem-solving in children. Tesis doctoral inedita. Harvard University, marzo $198 \mathrm{I}$.

GAI.TON, M.; SIMON, B., y CRol.1, P.: Inside the primary classroom. Boston: Routledge y Kegan Paul, 1980. INAGAK1, K: "Facilitation of knowledge integration through classtoom discussion". The Quarterly Newsletter of the Laboratory of Comparative Human Cognition (UCSD), april 1981, 3, (3), 26-28.

IN $\Lambda$ S $\Lambda \mathrm{KI}, \mathrm{K}$., y HATAN(), G.: "Motivational influences on epistemic observation». Japanese Journal of Educational Psychology, 1968, 16, 191-202.

INAGAKI, K., y HATANO, G.: "Amplification of cognitive motivation and its effects on epistemic observation". American Educational Research Journal, 1977, 14, 48, 591.

INHI:IDER, B., y PIAGil:T, J:: The grousth of logical thingking from childhood to adolescence. Nueva lork, Basic, 1958. Kimile, B.: "One child one teacher, one classroom; The story on one piece of writing". Language -1ris, $1980,57,680-693$. 
Kolitsov, V. A.: "Experimental study of cognitive activity in communication (with specific reference to concept formation)m. Soiviet Psychology, 1978, $1,2,23-38$.

Kins, D., y Ho, V.: "Self-directed acrivity and cognitive devélopment". Journal of Applied Development Psyrbology, I, (2), 1980, 119-133.

HL'H., D., y PHFI.PS, F.: "A methodology for observing development of a formal reasoning strategy". New Directions for Child Development, 1979 ( 9 ), 45-\$8.

KUHN, D., y PHELPS, E.: "The development of problem-solving strategies». En H. Reese y L. P. Lipsett (Eds.), Aduances in cbild development, vol. 17, N. Y. Academic Press, en prensa.

LAWIFR, $J$ : "Collectivity and individuality in Soviet educational theory". Contemporary Educational Psychology, 1980, S, 163-174.

LFoNTIF, A. N.: "The problem of activity in psycholugy". Fn J. V. Wertsch (F.d.), The concept of artivity in Soriet psychology. Armonk, N. Y.: M. F. Sharpe, $1981,37-7 \mathrm{I}$.

I.EONTIF, A. N., y LLRIA, A. R.: "The psychological ideas of L. S. Vygotski». En B. B. Wolman (Fd.), Historical roots of contemporary psychology'. Nueva York, Harper \& Row, 1968.

ILEVINA, R. F.: «L. S. Vygoiski ideas about the planning function of speech children». Fn J. V. Wertsch (Ed.). The concept of activity in Sotiet psychology. White Plains, M. Sharpe, 1981, págs. 279-299.

Lomox, B. F.: "Psychological processes and communication». Soviet Psychology, 1978, 17, 3-22.

Meman, H.: Learning Lessons. Cambridge: Harvard University Press, 1979.

Mén., H., y RıEı., M. M.: "Teachers "and students" instructional strategies». En L. Adler (F.d.), Issues in cross-cultural research, Nueva York, Acadcmic Press, en prensa.

Mligi, G., y DoISE, W': "Socio-cognitive conflict and structure of individual and collective perfomances». European Journal of Social Psychology, 1978, 8, 101-192.

PARTEN, M.: "Social participation among preschool children". Journal of Abnormal and Social Psycholog". $1932,27,243-269$.

PL:RRIT-CILR.MONT, A. N.: La construction de l'intelligence dans l'interaction sociale. Berna, Peter Lang, I979, Trad. cast. Aprendizaje-Visor (en prensa).

PlaceT, J.: "Piaget's theory». Fin P. H. Mussen (F.d.), Carmichael's Manual of Child Psychology (3.2 ed., rol. ii), Nueva York, Willey, 1970, 703-732..

SIIARAN, S.: "Cooperative learning in small groups: Recent methods and effects on achievement, attitudes, and ethnie relations", Kerien' of Educational Research, 1980, 50, 24 1-271.

Simon, B. (Ed.): Psychology in the Soliet Union. Stanford, California, Stanford University Press, 1957.

SIMON, B., y Simon, J. (Fds.): Educational psychology in the USSR, Stanford, California, Stanford University Press, 1963.

SI.ANINA, L. S.: "Specific features of the intellectual work of unsuccessful pupils». En B. Simon (Ed.), Psychology in the Soriet Union. Stanford University Press, 1957, $205-212$.

Vrgotski, L. S.: Mind in society. M. Cole, V. John-Steiner, S. Scribner y E. Souberman (Eds.), Cambridge, Harvard University Press, 1978.

VYGotski, L. S.: "The genesis of higher mental functions", en J. V. Wertsch (Ed.), The concept of activity in Soyiet Psychology. White Plains, M. Sharpe, en prensa.

WERTSCH, J. V.: "Adult-child interaction and the roots of metacognition». The Quarterly Newsletter on the Institute for Comparative Human Development. Rockfeller University, 1978, 2, (1), $19-18$.

W'ERTSCH, J. V.: "The regulation of human action and the given-new organiztion of private speech". En G. Zivin (Ed.), The deselopment of self-regulation through private speech. Nueva York, W'iley, 1979, 79-78.

WERTSCH, J. V. (Ed.): The concept of activity in Soviet Psychology, White Plains, M. Sharpe, 1981.

WERTSCH, J. V., y STONE, C. A.: «Microgenesis as a tool for development analysis». Quarterly Newsletter of the Laboratory of Comparative Human Development (UCSD), 1978, I, (1).

WOOD, D.; BRLNER, J. S., y ROSS, G.: "The role of tutoring in problem-solving". Journal of Child Psychology and Psychiatry, 1976, 17, 89-100. 